\title{
Bronchus-Ca: Mögliche EGFR-Mutation als Prädiktor
}

\author{
Subgruppenanalyse von LUX-Lung 1 zeigt Vervierfachung des PFS
}

Das Lungenkarzinom ist insbesondere in den fortgeschrittenen Stadien kaum bis gar nicht behandelbar. Die zumeist späte Entdeckung dieser Krebserkrankung führt daher zu einer hohen Mortalitätsrate. Zurzeit existiert keine zugelassene Therapie für Patienten mit fortgeschrittenem Lungenkarzinom, die nach Chemotherapie und einer Behandlung mit EGFR-TKI in ihrer Erkrankung fortgeschritten sind. Die Analyse der Studienergebnisse von LUX-Lung 1 weckt für eine bestimmte Subgruppe von Lungenkarzinompatienten Hoffnungen: Bei jenen Patienten, deren Tumoren wahrscheinlich EGFR (Epidermal Growth Factor Receptor)-Mutationen aufweisen, konnte der Tyrosinkinaseinhibitor Afatinib eine Vervierfachung des progressionsfreien Überlebens (PFS) verglichen mit Placebo erreichen.

Damit wurde eine signifikante Verlängerung der Zeit bis zum erneuten Tumorwachstum erzielt. Zusätzlich wies diese Patientensubgruppe einen Trend in Richtung eines verlängerten Gesamtüberlebens (OS) auf. Die aktualisierte post-hocAnalyse zu Afatinib aus der klinischen Phase III-Studie LUX-Lung 1 wurde beim Chicago Multidisciplinary Symposium in Thoracic Oncology, USA, präsentiert (1). Diese neue Analyse aktualisiert die ersten Ergebnisse der LUX-Lung 1-Studie, die vor kurzem auf dem 35. Kongress der European Society for Medical Oncology (ESMO) in Mailand vorgestellt wurden (2) und zunächst keine Verlängerung des Gesamtüberlebens gezeigt hatte.

Afatinib ist ein oraler Tyrosinkinaseinhibitor (TKI) der nächsten Generation. Im Gegensatz zu TKI der ersten Generation bindet Afatinib irreversibel sowohl an EGFR (Epidermal Growth Factor Receptor) als auch HER2 (Human Epidermal Growth Factor Receptor 2) (3). Die Phase IIb/III-Studie LUX-Lung 1 verglich Afatinib $^{1}$ mit Placebo an Patienten mit fortgeschrittenem NSCLC, deren Krankheit nach Chemotherapie und einer Behandlung mit einem EGFR-TKI der ersten Generation (Gefitinib oder Erlotinib) fortgeschritten war.

Die vorgestellte Subgruppenanalyse umfasste zwei Drittel aller Patienten aus der Studie (391 von 585 Patienten). Diese Patientengruppe wies aufgrund der ermittelten klinischen Kriterien, wie Ansprechrate und Therapiedauer der vorangegangenen EGFR-TKI Behandlung mit hoher Wahrscheinlichkeit EGFR-Mutationen auf.

Die beim ESMO in Mailand präsentierte Ergebnisse zeigten, dass Afatinib die Zeit bis zur Tumorprogression in allen Patientensubgruppen signifikant verlängerte; das progressionsfreie Überleben betrug 3,3 Monate gegenüber 1,1 Monate mit Placebo. Mit Afatinib behandelte Patienten wiesen auch eine signifikant höhere Rate in der Tumorkontrolle oder Tumorverkleinerung auf (Disease Control Rate; DCR: 58 Prozent), als Patienten unter Placebo (DCR: 19 Prozent). Beobachtet wurde mit Afatinib auch eine signifikante Verbesserung der zentralen Symptome, die mit Bronchialkarzinomen verbundenen sind wie Husten, Atemnot (Dyspnoe) und Schmerzen; außerdem verzögerte Afatinib signifikant die Zeit bis zur Verschlechterung von Husten, Dyspnoe und Brustschmerz.

Die wesentlichen, unerwünschten Arzneimittelwirkungen unter Afatinib entsprechen den bisherigen Erkenntnissen und sind im wesentlichen Diarrhö und Hautausschlag.

Für das Krankheitsmanagement bestätigen die Ergebnisse der Subgruppenanalyse den Weg hin zu einer personalisierten Therapie, bei welcher gezielt auf die Patientencharakteristika eingegangen wird, um den besten Therapieerfolg zu erzielen.

Quelle: Presseaussendung Boehringer Ingelheim

Afatinib (BIBW 2992) befindet sich derzeit in der klinischen Erprobung. Die Wirksamkeit und Sicherheit ist nicht vollständig erforscht.

\section{LITERATUR}

1 Miller et al. (2010) Subgroup analysis of LUXLung 1: A randomized Phase III Trial of Afatinib (BIBW 2992) + Best Supportive Care (BSC) versus Placebo + BSC in Patients with NSCLC Failing 1-2 Lines of Chemotherapy and Erlotinib or Gefitinib. Oral presentation at Chicago Multidisciplinary Symposium in Thoracic Oncology, Chicago, USA

2. Miller et al. (2010) Phase $\mathrm{llb} / \mathrm{lll}$ double-blind randomized trial of BIBW 2992, an irreversible inhibitor of EGFR/HER1 and HER2 + best supportive care (BSC) versus placebo + BSC in patients with NSCLC failing 1-2 lines of chemotherapy and erlotinib or gefitinib (LUX-Lung 1). Oral presentation at The European Society of Medical Oncology (ESMO) annual meeting, Milan. Abstract ID: LBA1

3. Li D et al. (2008) BIBW2992, an irreversible EGFR/HER2 inhibitor highly effective in preclinical lung cancer models. Oncogene, 27, pp.4702-4711 Schmerzen; außerdem verzögerte Afatinib signifikant die Zeit bis zur Verschlechterung von Husten, Dyspnoe und Brustschmerz.

- Gegenüber den bisherigen Studien wurden keine neuen unerwünschten Ereignisse unter Afatinib beobachtet; die wesentlichen, unerwünschten Arzneimittelwirkungen waren Durchfall (Diarrhö) und Hautausschlag.

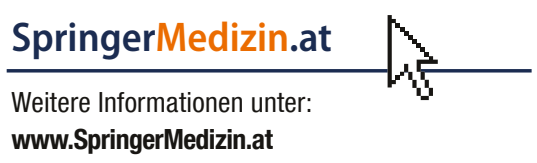

